\title{
Reseña del libro: "Momentos y procesos de la investigación en educación ambiental"
}

\section{María Magdalena Castorena Dorado}

Universidad Pedagógica Nacional -Ajusco

\begin{abstract}
La inteligencia consiste no solo en el conocimiento, sino también en la destreza de aplicar los conocimientos en la práctica
\end{abstract}

Aristóteles

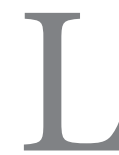

a educación ambiental es un tema relevante, porque todos somos responsables cuando sobreviene una crisis por la excesiva explotación de los recursos naturales, cuyas consecuencias son la pérdida de biodiversidad, destrucción de la capa de ozono, lluvia ácida, cambio climático, modificación atmosférica, desertificación por erosión, entre otros; de ahí el interés de los jóvenes investigadores por iniciar en el campo de la educación ambiental, donde se requiere de dedicación y compromiso, por ello, el propósito de este texto es mostrar los momentos y observar los procesos en el ámbito de la investigación en educación ambiental.

En los últimos años organismos internacionales, instituciones educativas y grupos sociales han enfatizado la falta de una Educación Ambiental que contribuya, a través de la construcción de una cultura ambiental, a una nueva racionalidad para promover e impulsar alternativas que permitan enfrentar los diversos problemas ambientales que nos aquejan en nuestro entorno (Arias, 2009), para ello se considera llevar a cabo una revisión de los acuerdos, estrategias y programas como los de Estocolmo, 1972; Belgrado, 1975 y Tbilisi, 1977; fundamentalmente con el interés de reconstruir el enfoque educativo de la Educación Ambiental propuesto por los participantes en las reuniones anteriormente citadas, un enfoque humanista integrando la diversidad, rescatando prácticas para trasmitir la constitución de una sociedad más justa, humana, equitativa y respetuosa de la naturaleza, cabe señalar que se habla de un sujeto emancipado, reflexivo consciente participativo, responsable, solidario cooperativo, etc. (Terrón, 2004). 
La Organización de las Naciones Unidas para la Educación, la Ciencia y la Cultura, recomienda a los países del mundo impulsar la Educación Ambiental como un proyecto civilizatorio distinto, para transitar hacia un cambio social planetario en el que todos los habitantes logren asir una cultura ambiental, como parte de su proyecto de vida personal, comunitario y sociocultural (UnEsco, 1977).

La educación ambiental ha sido un campo fértil para la investigación educativa, un campo emergente de reflexión y de propuestas teóricas, las cuales se traducen en acciones analíticas, críticas y reflexivas. En la actualidad es un campo real de teoría, investigación y práctica, con una breve historia, pero con múltiples vertientes en los distintos ámbitos de la educación.

El libro "Momentos y procesos de la investigación en educación ambiental", se conforma de seis capítulos en los cuales el autor plantea dar acompañamiento al investigador principiante llevándolo de la mano para la construcción de su propio trabajo, el autor de este libro, cuenta con la facilidad y la didáctica de explicar cada una de las características que debemos considerar dentro de la investigación en educación ambiental, el libro cuenta con dos anexos los cuales ayudan a enriquecer la construcción del proyecto, apropiándose cada vez más de su tema de investigación.

El primer capítulo, muestra un panorama general sobre la investigación en educación ambiental, comprendiendo desde la definición, postura, trayectoria e influencia que ha tenido a partir de las reuniones internacionales, que han sido el sustento y la dificultad de su desarrollo y consolidación ante la coexistencia de diferentes posturas.

El segundo capítulo, ofrece elementos básicos para comprender la naturaleza y alcance que ha tenido la educación ambiental, considerando esta información como el marco general con particularidades específicas del tema investigado, así mismo desarrollando estrategias y pensamientos reflexivos.

En el tercer capítulo, se presenta una serie de aspectos básicos para tomar decisiones con respecto a la postura paradigmática que llevará la investigación, como nos señala Gaudiano (1994) simplemente proporciona herramientas teóricas para analizar y formular el proyecto de educación ambiental, contrastando diferentes tradiciones intelectuales: el positivismo, interpretativo y crítico, con el fin de ilustrar su función en la explicación de la realidad. Hace un llamamiento para desarrollar una teoría de la educación ambiental propia en América Latina y el Caribe, y sugiere algunas pautas de análisis de los proyectos.

El cuarto capítulo va encaminado a la perspectiva metodológica de la investigación en la educación ambiental, se presenta el modo de ver el problema, no como el conjunto rígido de reglas (House, 1985); se mencionan varios tipos de perspectivas como cuantitativa, cualitativa, mixta, investigación acción y compleja; cada una de ellas cuenta con su supuesto epistemológico y ofrecen resultados relevantes en el campo del educación ambiental.

En el quinto capítulo, se abordan los tipos de investigación en educación ambiental, cabe señalar que siendo uno de los 
conflictos por los que pasan los jóvenes investigadores al elegir el tipo de práctica que deberán contemplar en el desafío de la futura interacción con la investigación de la educación ambiental, el escritor propone una tipología de investigación de acuerdo con los criterios de autores como: González Gaudiano (1997), Sauvé (2000), Caridde y Meira (2001), entre otros, proporcionando información para delimitar el proceder metodológico, de una manera más sencilla, finalmente dentro de este apartado es preciso señalar que "De acuerdo con la perspectiva en que se desarrolla la investigación deben tomarse decisiones previas como la delimitación del objeto a investigar la forma de concebir la producción del conocimiento...la concepción de la propia actividad de la investigación" (Calixo, 2015:120).

Del protocolo al proyecto de investigación, es el título del capítulo sexto, es en sí la forma más didáctica de mostrar la construcción del objeto de estudio, objetivos e hipótesis, la justificación, marco teórico, el proceso metodológico, referencias y anexos; cada uno de estos elementos se describen a partir de puntos clave facilitando la creación de su propio proyecto de investigación, no debemos olvidar que a partir de la construcción del objeto de estudio, en la investigación se tendrá claridad con respecto a donde queremos llegar y al delimitar el objeto de estudio dentro de este apartado podremos encontrar, como uno de sus contenidos, una tabla en la cual se encuentran sugerencias con respecto a los verbos a utilizar y así proceder a la elaboración de la hipótesis, siendo aquellos elementos que serán medidos en una persona, grupo u objeto; el supuesto dentro de este trabajo debemos tomarlo en cuenta ya que es la conjetura que orienta el desarrollo de la investigación, todo esto sustentado a partir del marco teórico con el apoyo de las investigaciones realizadas que se relacionan con el tema.

Dentro del protocolo nos menciona que el proceder metodológico es un apartado más que contemplar: técnicas e instrumentos y estrategias para llevar a cabo la realización de los instrumentos, es preciso señalar como lo menciona el autor, la observación, encuesta y entrevista que son de gran utilidad para la obtención de resultados formando parte clave dentro del protocolo de investigación y analizarlos de manera cuantitativa y cualitativa, y, finalmente, las referencias documentales utilizadas a lo largo de la investigación.

Sus doscientas veinte páginas, seis capítulos y dos anexos que conforman el libro "Momentos y procesos de la Investigación en educación ambiental", son de gran ayuda para aquel joven investigador que inicia un acercamiento a la Investigación en Educación Ambiental, y que mejor acompañado de este material, que brinda las herramientas necesarias para recorrer el camino de la investigación hasta conformar su protocolo de investigación, con bases teóricas que sustentan cada uno de los argumentos planteados.

En cada uno de los capítulos se fomenta el compromiso, la reflexión y dedicación hacia la construcción y trasformación de nuestros saberes. Morín (2004) nos diría que propone un nuevo tipo de conciencia 
que se le denomina planetaria ya que cuando se adquiere esta conciencia se fomenta la capacidad de analizar y reflexionar sobre los diferentes tipos de trabajo que realice el investigador, lo que es sin duda una gran labor.

Finalmente, es necesario agradecer al autor de este libro titulado "Momentos y procesos de la investigación en educación ambiental" por el tipo de material que nos ofrece, el cual tiene como objetivo principal brindar la ruta hacia el camino de la investigación en educación ambiental.

\section{Bibliografía}

Calixto, R. (2011) Educación popular ambiental. Revista Trayectorias, Vol. XII, Núm. 30, 24-39. en: trayectorias.uanl.mx/30/pdf/educacion_popular_ambiental. pdf,

Calixto, R. (2015) Momentos y procesos de la investigación en educación ambiental. México. Universidad Pedagógica Nacional.

González, E. (1999) Otra lectura a la historia de la Educación Ambiental en América Latina y el Caribe. Antología del Proyecto Educación Ambiental para la
Amazonia. Tópicos en Educación Ambiental. 1 (1): 9-26.

González, E. y Arias, M. Á. (2011) La educación ambiental institucionalizada: proceso, reflexiones y posibilidad en: Calixto, R., García, Mayra y Gutiérrez Daniel. Educación e investigación ambientales y sustentabilidad. México. UPN. 181-226.

González, E. y Bravo T. (2008) Estado del conocimiento de la investigación en educación ambiental en México. Avances y desafíos. en: Felipe Reyes y Teresa Bravo (coordinadores). Educación ambiental para la sustentabilidad en México. UNICACH,168-186, recuperado el 01/06/2014 en: http://anea.org.mx/docs/ EdAmbSustentabilidadMexico.pdf.

Hernández, R. et al (2006) Metodología de la Investigación. Mc Graw-Hill. México

Morin, E. (2004) Tierra patria, Buenos Aires.

Sauvé L. (1999) La educación ambiental entre la modernidad y la posmodernidad, en busca de un marco de referencia integrador. Revista Tópicos de Educación Ambiental. 1 (2), 7-25 recuperado el 01/05/2014 en: www.ambiente.gov.ar/infotecaea/descargas/sauve02.pdf.

Terrón, E. (2004) La educación ambiental en la educación básica, un proyecto inconcluso. Revista Latinoamericana de Estudios Educativos. México. Recuperado el 21 de septiembre de 2015 en:<http://oai.redalyc.org/ articulo.oa? id=27034404 $>$ ISSN 0185-1284

UNESCO (1980) La educación ambiental. Las grandes orientaciones de la conferencia del Tbilisi. París. Francia. 